\title{
Icariin regulates the proliferation and apoptosis of human ovarian cancer cells through microRNA-21 by targeting PTEN, RECK and Bcl-2
}

\author{
JINGWEI LI, KAILEI JIANG and FUJIE ZHAO \\ Department of Obstetrics and Gynecology, Shengjing Hospital of China Medical University, \\ Shenyang, Liaoning 100004, P.R. China
}

Received January 20, 2015; Accepted March 5, 2015

DOI: 10.3892/or.2015.3891

\begin{abstract}
Icariin is the main active ingredient found in the traditional Chinese medicinal plant Epimedium, and exhibits various pharmacological effects such as enhanced immune function, anticancer activity, improved cardiovascular function and endocrine adjustment. However, the effect of icariin on ovarian cancer and the related mechanism have never been investigated. In the present study, we aimed to verify whether icariin inhibits the proliferation and increases the apoptosis of human ovarian cancer cells, and its molecular mechanism in order to establish an association and identify potential therapeutic targets. In the present study, ovarian cancer A2780 cells were treated with various concentrations of icariin, and the cell viability was evaluated by 3,3-(4,5-dimethylthiazol2-yl)-2,5-diphenyltetrazolium bromide (MTT) assay. Flow cytometry and caspase- 3 colorimetric assay were performed to observe apoptotic changes in the A2780 cells. qPCR analysis was used to analyze miR-21 expression in the A2780 cells. Western blot analysis was used to assess PTEN, RECK and Bcl-2 protein expression. Transfection of microRNA-21 (miR21) and anti-miR-21 was used to investigate expression of its target genes associated with cell proliferation and apoptosis. Icariin concomitantly suppressed cell proliferation, accelerated apoptosis and increased caspase-3 activity in the A2780 cells. In the ovarian cancer A2780 cells, icariin substantially decreased the miR-21 expression level, increased PTEN and RECK protein expression levels and decreased the Bcl-2 protein expression level. Notably, miR-21 regulated the potential anticancer effects of icariin on cell proliferation and apoptosis by targeting PTEN, RECK and Bcl-2 in the ovarian
\end{abstract}

Correspondence to: Dr Fujie Zhao, Department of Obstetrics and Gynecology, Shengjing Hospital of China Medical University, 36 Sanhao Street, Heping, Shenyang, Liaoning 100004, P.R. China E-mail: fujiezhaohj@163.com

Key words: icariin, ovarian cancer, microRNA-21, PTEN, RECK, Bcl-2 cancer A2780 cells. Our results demonstrated that icariin is an excellent candidate antitumor agent which exhibits an anticancer curative effect on ovarian cancer cells. miR-21 and its target genes may play a vital role in the molecular mechanism of the anticancer effects of icariin.

\section{Introduction}

Ovarian cancer incidence ranks third among all gynecological tumors after cervical and uterine cancer, while it is the most lethal (1). Early symptoms of ovarian cancer are occult, and it is quite difficult to identify the tissue types and whether the tumor is benign or malignant. Moreover, more than $70 \%$ of ovarian cancer patients are diagnosed at an advanced stage limiting treatment options (2). Statistics show that the 5-year survival rate of FIGO I or II ovarian cancer patients is $70-90 \%$, when many patients can be cured only by surgery, while the 5 -year survival rate of FIGO III or IV patients is only $20 \%$ (3). Ovarian cancer is a malignant tumor that seriously threaten the health of women, and the incidence of ovarian cancer is increasing yearly.

MicroRNAs (miRs) are a type of small-molecule endogenous RNAs, which play an important regulatory role in the growth process and the development of higher organisms (4). miR-21 is a member of the miRs and is highly expressed in various human tumors where it participates in cell proliferation, differentiation and apoptosis, and is closely related to the growth, invasion and metastasis of tumors (5). Chan et al found that suppression of miR-21 accelerated the apoptosis and increased the chemosensitivity of ovarian cancer (6). Liu et al reported that berberine sensitized ovarian cancer cells to cisplatin through inhibiting miR-21 expression (7).

PTEN/MMAC1/TEP1 is a tumor-suppressor gene similar to $\mathrm{pRb}$ and $\mathrm{p} 53$, and has attracted much attention. It has been demonstrated that deficiency of the PTEN gene and protein expression abnormalities appear in a variety of malignant tumor tissues and tumor cell lines (8). Wu et al demonstrated that overexpression of PTEN improved the cisplatin-resistance of human ovarian cancer cells (9). Lau et al reported that E-cadherin inhibited ovarian cancer cell growth via $\beta$-cateninEgrl-mediated PTEN expression (10). Lou et al demonstrated that miR-21 promoted the cell proliferation, invasion and 
migration abilities of ovarian epithelial cancer cells by inhibiting PTEN protein expression (11).

RECK is a newly discovered tumor-suppressor gene. RECK protein has a unique inhibitory effect on the expression and activity of MMPs, as a matrix metalloproteinase inhibitor, and RECK gene expression is closely related to tumor invasion, metastasis and angiogenesis (12). Studies have shown that RECK gene expression in liver (13), pancreatic (14), breast (15) and lung cancer (16) is negatively correlated with tumor invasiveness, and patients with high RECK gene expression have a significantly more favorable prognosis than those with low expression. Further research reported that upregulation of RECK reduced ovarian cancer amplification (17). Histone deacetylase inhibitor inhibited human ovarian cancer cell migration through upregulation of RECK and downregulation of matrix metalloproteinase-2 (MMP-2) in HDAC4-blocked SKOV-3 cells (18).

The relationship between apoptosis and tumors is one of the 'hotspots' in recent years. Uncontrolled apoptosis is an important feature leading to tumor occurrence and development. The Bcl-2 family has attracted increased attention, and Bcl-2 and Bax are important members of this family. They are mainly involved in the regulation of apoptosis through the mitochondrial pathway (19). Xie et al found that estrogen and progesterone significantly inhibited the cell survival and promoted the cell apoptosis of ovarian cancer cells through inhibition of the expression of Bcl-2 (20). Ma et al showed that a specific cell-penetrating peptide induced apoptosis by downregulation of Bcl-2 in human ovarian cancer SKOV3 cells (21).

Icariin, a plant flavonoid glycoside, is an effective pharmacological ingredient found in the traditional Chinese medicinal plant Epimedium (22). Due to its estrogen-like structure, icariin has a wide range of pharmacological effects on the heart and brain vascular system, bone metabolism, immune and nervous system, sexual function, and exhibits anti-inflammatory and antitumor effects (23). Wang et al found that icariin decreased the viability and induced the apoptosis of MLTC- 1 cells through regulation of the expression of Bcl-2/Bax (24). It is vital to explore new prospective effective anticancer drugs and develop therapies against ovarian cancer. The present study aimed to investigate the anticancer effect of icariin against ovarian cancer cells. We assessed whether icariin regulates the proliferation and apoptosis of human ovarian cancer cells and further explored the mechanism underlying its activity.

\section{Materials and methods}

Chemicals and reagents. The chemical structure of icariin (with a purity $\geq 94 \%$; Sigma) is shown in Fig. 1. Roswell Park Memorial Institute (RPMI)-1640 and fetal calf serum (FBS) were purchased from Gibco HyClone (Invitrogen Co., South America) and Invitrogen Co., BRL, respectively. 3,3-(4,5-Dimethylthiazol-2yl)-2,5diphenyltetrazolium bromide (MTT) was purchased from Sangon Biotech (Shanghai, China). Annexin V-FITC/propidium iodide (PI) apoptosis detection kit was obtained from Takara Bio, Inc. (Dalian, China). Caspase-3 colorimetric assay kits were obtained from Beyotime (Nanjing, China). TRIzol reagent, cDNA synthesis kit and SYBR-Green kit were obtained from Tiangen (Beijing, China). Lipofectamine 2000 and

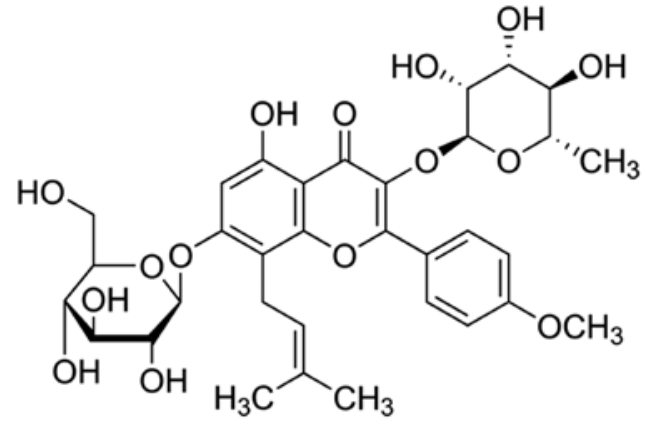

Figure 1. Chemical structure of icariin.

Opti-MEM I were obtained from Invitrogen Life Technologies. The BSA kit was obtained from Thermo Scientific, USA.

Cell lines and culture. The human ovarian cancer A2780 cell line was acquired from the Animal Laboratory of Shengjing Hospital Affiliated to China Medical University. The A2780 cells were cultured in RPMI-1640 containing 10\% FBS with $100 \mathrm{U} / \mathrm{ml}$ penicillin and $100 \mathrm{U} / \mathrm{ml}$ streptomycin and were maintained at $37^{\circ} \mathrm{C}$ in a humidified atmosphere of $5 \% \mathrm{CO}_{2}$. A2780 cells were passaged every 2-3 days.

Cell viability assay. A2780 cells ( $5 \times 10^{3} /$ well) were seeded into 96-well culture plates and treated with icariin $(3,6,13,25,50$ and $100 \mu \mathrm{M})$, or vehicle for 24,48 or $72 \mathrm{~h}$. MTT (10 $\mu \mathrm{l})$ was added to the A2780 cells and incubation was carried out for $4 \mathrm{~h}$ at room temperature. The supernatant was discarded, and dimethyl sulfoxide (DMSO) was added to each well and was shaken for $20 \mathrm{~min}$. Then, A2780 cell viability was determined using the MTT assay.

Determination of cell apoptosis by flow cytometry. A2780 cells $\left(2 \times 10^{6} /\right.$ well $)$ were seeded into 6-well culture plates and treated with icariin $(13,25$ and $50 \mu \mathrm{M})$, or vehicle for 24 , 48 or $72 \mathrm{~h}$. A2780 cells were collected and washed twice with ice-cold phosphate-buffered saline (PBS). A2780 cells $\left(1 \times 10^{6} / \mathrm{ml}\right)$ were resuspended with Annexin $\mathrm{V}$ binding buffer. Annexin V-FITC $(5 \mu \mathrm{l})$ was added to the A2780 cells and stained for $30 \mathrm{~min}$ at darkness. Then, $10 \mu \mathrm{l} \mathrm{PI}$ was added to the A2780 cells and stained for 10 min according to the manufacture's instructions (Takara Bio, Inc.). Cell apoptosis of A2780 cells was immediately detected using flow cytometry (EPICS ${ }^{\circledR}$ Altra $^{\mathrm{TM}}$; Olympus).

Detection of caspase-3 activity. A2780 cells ( $2 \times 10^{6} /$ well) were seeded into 6-well culture plates and treated with icariin (13, 25 and $50 \mu \mathrm{M}$ ), or vehicle for 24,48 or $72 \mathrm{~h}$. The caspase- 3 activity in fluorescence was detected at the wavelength of $405 \mathrm{~nm}$ with the caspase- 3 colorimetric assay kits.

qPCR analysis of miR-21 expression. A2780 cells ( $2 \times 10^{6} /$ well) were seeded into 6-well culture plates and treated with icariin $(13,25$ and $50 \mu \mathrm{M})$, or vehicle for 24,48 or $72 \mathrm{~h}$. According to the manufacturer's instructions (Tiangen), total RNA was isolated from the A2780 cell samples using TRIzol reagent. cDNAs were synthesized and detected using cDNA synthesis kit, according to the manufacturer's instructions (Tiangen). 


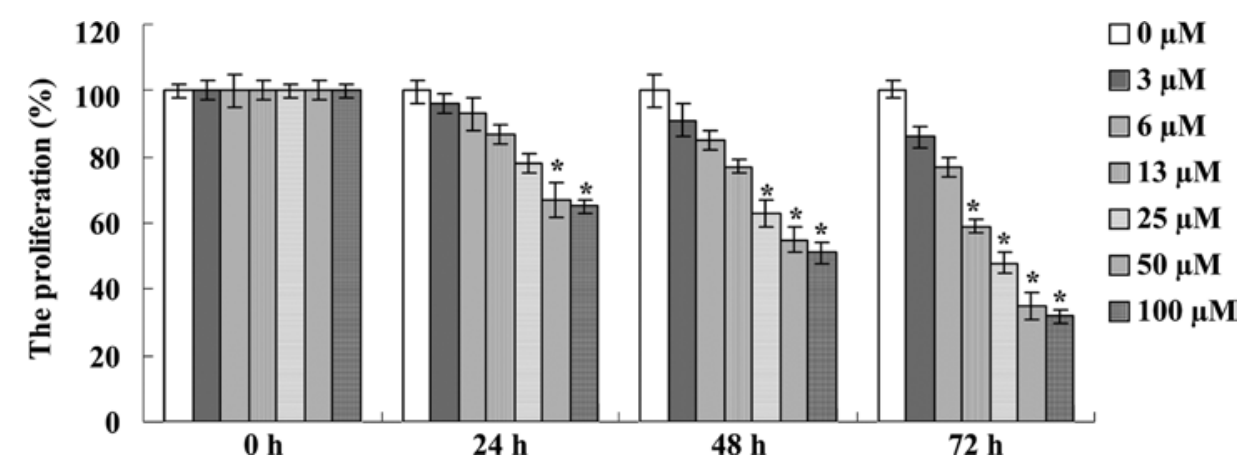

Figure 2. Icariin suppresses the proliferation of ovarian cancer cells. ${ }^{*} \mathrm{p}<0.01$ compared with $0 \mu \mathrm{M}$ icariin-treatment group.

The expression of miR-130b was detected using the SYBRGreen kit.

Immunoblot analysis. A2780 cells (2x10\% into 6-well culture plates and treated with icariin $(13,25$ and $50 \mu \mathrm{M}$ ), or vehicle for 24,48 or $72 \mathrm{~h}$. A2780 cells were harvested and washed with ice-cold PBS. The harvested cells were suspended in lysis buffer (10 mM Tris, $150 \mathrm{mM} \mathrm{NaCl}, 1 \%$ Triton X-100, 1\% Na-deoxycholate, 5 mM EDTA) and placed on ice for $30 \mathrm{~min}$. A2780 cells were harvested and centrifuged at $12,000 \mathrm{x} \mathrm{g}$ for $10 \mathrm{~min}$ at $4^{\circ} \mathrm{C}$. The total proteins of the cell lysates were determined using the BSA kit (Thermo Scientific). Equal protein was separated by SDS-PAGE, and then transferred onto PVDF membranes (0.22 mm; Millipore, Billerica, MA, USA). The membranes were blocked and incubated overnight with anti-PTEN (1:1,000), anti-PTEN (1:1,000), antiBcl-2 $(1: 1,500)$ and anti- $\beta$-actin $(1: 3,000)$ (all from Santa Cruz Biotechnology, Inc., Santa Cruz, CA, USA) overnight at $4^{\circ} \mathrm{C}$. The membranes were developed using ECL reagent $(1: 1,000$; Santa Cruz Biotechnology, Inc.) and imaged using a polaroid imaging system (Amersham).

Transfection of miR-21 and anti-miR-21. miR-21 inhibitors and miR-21 mimics were chemically synthesized by BeastBio Co., Ltd. (Shanghai, China). A2780 cells (2x10\%/well) were seeded into 6-well culture plates. miR-21 mimics (100 pmol) and miR-21 inhibitors were transfected into the A2780 cells with Lipofectamine 2000 and Opti-MEM I reduced serum medium (Invitrogen Life Technologies). The expression of miR-21 was detected using the SYBR-Green kit. The primers for miR-21 were: 5'-GCCCGCTAGCTTATCAGACTGATG-3' and 5'-GCCCGCTAGCTTATCAGACTGATG-3', respectively. The primers for U6 were: 5'-GCGCGTCGTGAAGCG TTC-3' and 5'-GTGCAGGGTCCGAGGT-3', respectively.

Statistical analysis. Each experiment was repeated at least three times. Data analysis was performed with SPSS 17.0 software (SPSS, Inc., Chicago, IL, USA), and data are shown as the mean $\pm \mathrm{SD}$. $\mathrm{P}<0.05$ was considered to indicate a statistically significant difference.

\section{Results}

Icariin suppresses the proliferation of ovarian cancer cells. To explore the potential anticancer effect of icariin on the proliferation of ovarian cancer A2780 cells, MTT assays were performed. As expected, icariin treatment clearly reduced the cell proliferation of the A2780 cells in a dose- and time-dependent manner in comparison with the $0 \mu \mathrm{M}$ icariin treatment group (Fig. 2). Particularly, treatment with icariin (50 and $100 \mu \mathrm{M})$ significantly inhibited the proliferation of A2780 cells at 24, 48 and 72 h, with statistical significance (Fig. 2). Meanwhile, treatment with $25 \mu \mathrm{M}$ of icariin for 48 and $72 \mathrm{~h}$ significantly reduced the proliferation of A2780 cells and treatment with $13 \mu \mathrm{M}$ of icariin for $72 \mathrm{~h}$ also significantly reduced the proliferation of A2780 cells (Fig. 2). Based on the MTT assay results, icariin treatments at final concentrations of 13,25 and $50 \mu \mathrm{M}$ at $48 \mathrm{~h}$ were chosen for future study.

Icariin promotes the cell apoptosis of ovarian cancer cells. In our study, we determined that icariin promoted the cell apoptosis of ovarian cancer A2780 cells. Thus, flow cytometry was used to measure the cell apoptosis of A2780 cells. Notably, a significant increase in cell apoptosis was observed following the icariin treatment at the final concentrations of 25 and $50 \mu \mathrm{M}$ for $48 \mathrm{~h}$ (Fig. 3A and B).

Icariin induces caspase-3 activity in ovarian cancer cells. To further investigate whether icariin induces caspase-3 activity of ovarian cancer A2780 cells, caspase-3 colorimetric assays were employed. Following treatment with icariin at concentrations of 25 and $50 \mu \mathrm{M}$ for $48 \mathrm{~h}$, the dose-dependent caspase-3 activity was obviously increased, when compared with the activity in the $0 \mu \mathrm{M}$ icariin treatment group (Fig. 4). These results clearly indicated that icariin efficiently induced apoptosis of the A2780 cells, and this apoptotic effect was exerted in a dose-dependent manner (Figs. 3 and 4).

Icariin suppresses miR-21 expression in ovarian cancer cells. To determine whether the expression of miR-21 is correlated with icariin treatment, we evaluated the expression level of miR-21 in the A2780 cells. As shown in Fig. 5, the miR-21 expression level in the A2780 cells was significantly decreased following treatment with icariin at concentrations of 25 and $50 \mu \mathrm{M}$ for $48 \mathrm{~h}$, compared with the $0 \mu \mathrm{M}$ icariin treatment group.

Icariin increases PTEN protein expression in ovarian cancer cells. We determined the expression of PTEN in the potential anticancer effect of icariin to study the role of miR-21 in ovarian cancer A2780 cells. As a result, icariin $(25$ and $50 \mu \mathrm{M})$ effectively increased PTEN protein expression in the A2780 

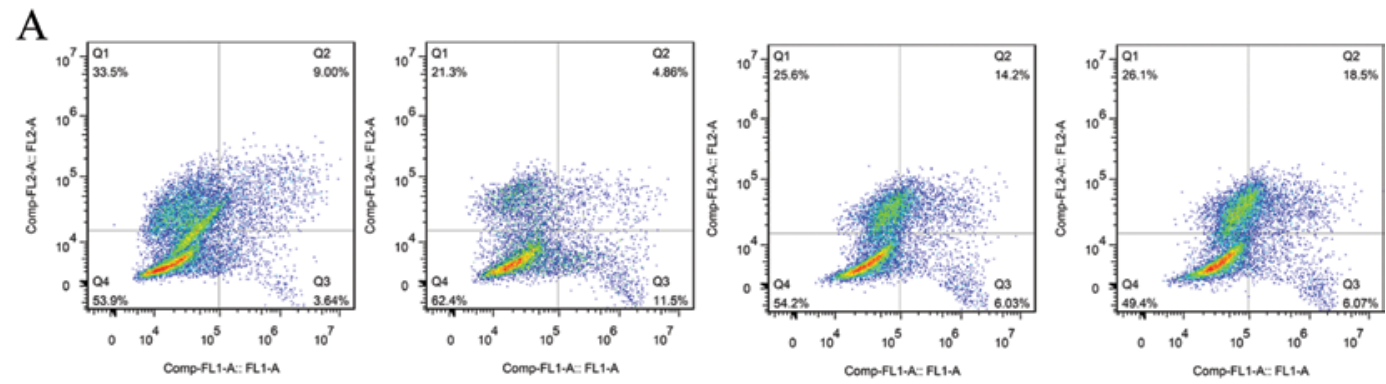

B

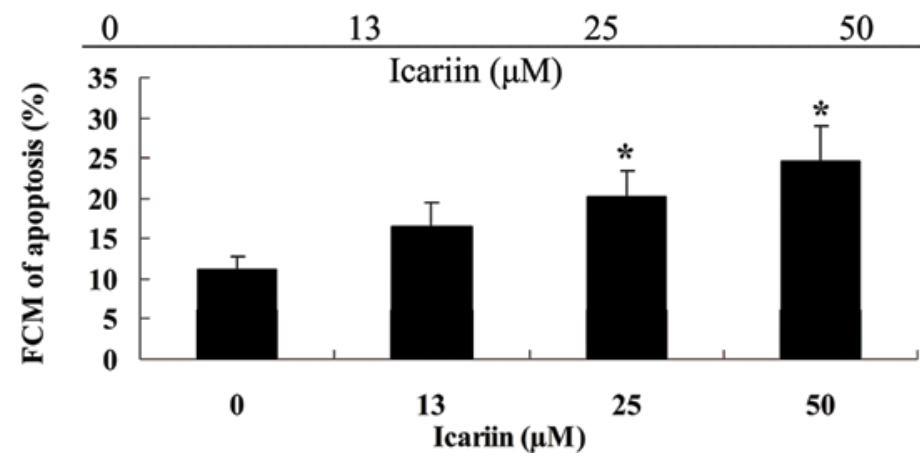

Figure 3. Icariin promotes the cell apoptosis of ovarian cancer cells. Icariin promoted the apoptosis of (A) A2780 cells. (B) Statistical analysis of the cell apoptosis level. " $\mathrm{p}<0.01$ compared with $0 \mu \mathrm{M}$ icariin-treatment group.

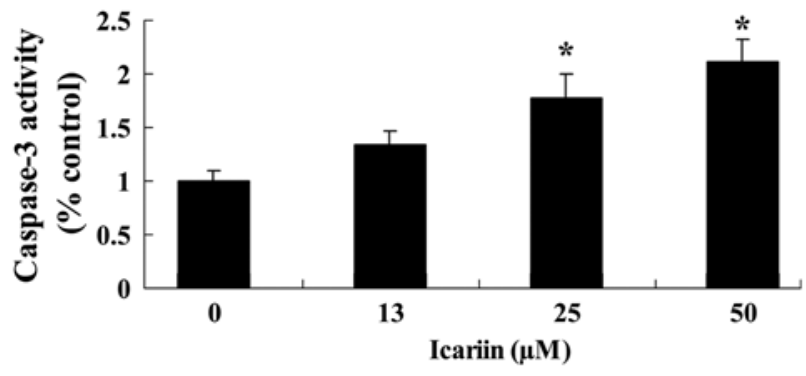

Figure 4. Icariin induces caspase-3 activity in the ovarian cancer cells. ${ }^{*} \mathrm{p}<0.01$ compared with the $0 \mu \mathrm{M}$ icariin-treatment group.

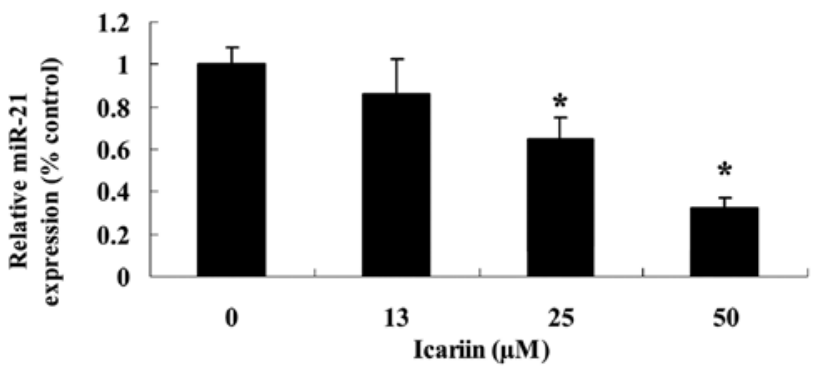

Figure 5. Icariin suppresses miR-21 expression in the ovarian cancer cells. " $\mathrm{p}<0.01$ compared with the $0 \mu \mathrm{M}$ icariin-treatment group.

cells at $48 \mathrm{~h}$, compared with that in the $0 \mu \mathrm{M}$ icariin treatment group (Fig. 6A and B).

Icariin increases RECK protein expression in ovarian cancer cells. To evaluate the potential efficacy of icariin treatment on RECK protein in the ovarian cancer A2780 cells, we detected
RECK protein expression levels. Following treatment with icariin at concentrations of 25 and $50 \mu \mathrm{M}$ for $48 \mathrm{~h}$, the RECK protein expression level was markedly increased in comparison to the level in the $0 \mu \mathrm{M}$ icariin treatment group (Fig. 7A and B).

Icariin suppresses $B c l-2$ protein expression in the ovarian cancer cells. To further investigate the potential effect of icariin on Bcl-2 protein expression in the ovarian cancer A2780 cells, the Bcl-2 protein expression level in the A2780 cells was measured. As shown in Fig. 8A and B, the Bcl-2 protein expression level was decreased following treatment with icariin at concentrations of 25 and $50 \mu \mathrm{M}$ for $48 \mathrm{~h}$, compared with that in the $0 \mu \mathrm{M}$ icariin treatment group.

Anti-miR-21 influences the expression levels of PTEN, RECK and Bcl-2 protein in the ovarian cancer cells. We selected miR-21 and its potential target proteins PTEN, RECK and Bcl-2 to study the effect of icariin on ovarian cancer A2780 cells. The miR-21 inhibitor was transfected with Lipofectamine 2000 into the A2780 cells. We found that miR-21 expression was effectively silenced in the A2780 cells, compared with the control group (Fig. 9A). In addition, PTEN and RECK protein expression levels were increased in the A2780 cells, compared with these levels in the control group (Fig. 9B). However, Bcl-2 protein expression level in the A2780 cells was reduced in comparison with the level in the control group (Fig. 9B).

Overexpression of miR-21 influences the effect of icariin on ovarian cancer cells. To further investigate the potential connection between overexpression of miR-21 and the potential effect of icariin on ovarian cancer A2780 cells, miR-21 mimics were transfected with Lipofectamine 2000 into the A2780 cells. Our results indicated that transfection of miR-21 
A

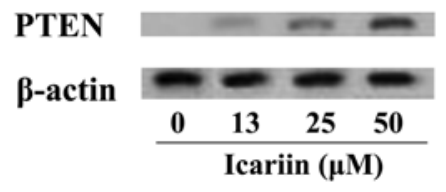

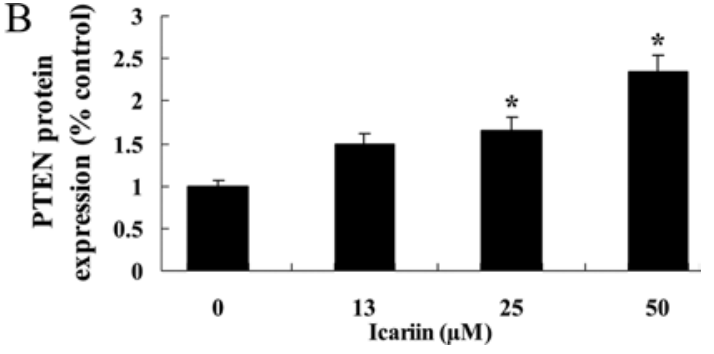

Figure 6. Icariin increases PTEN protein expression in the ovarian cancer cells. (A) The effects of icariin on PTEN by western blotting assays. (B) Statistical analysis of the PTEN protein level. " $\mathrm{p}<0.01$ compared with the $0 \mu \mathrm{M}$ icariin-treatment group.

A

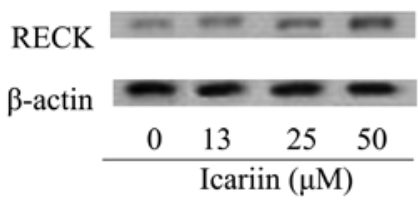

B

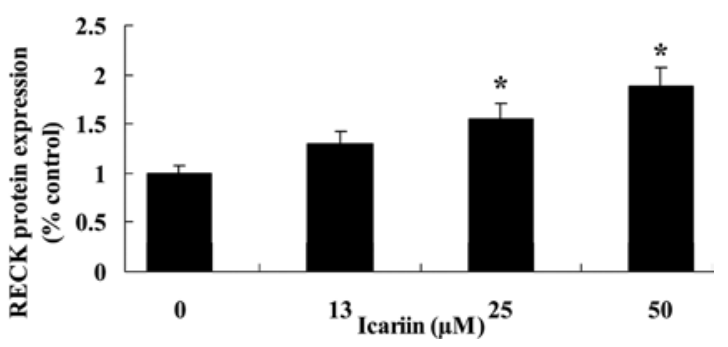

Figure 7. Icariin increases RECK protein expression in the ovarian cancer cells. (A) The effects of icariin on RECK by western blotting assays. (B) Statistical analysis of the RECK protein level. " $\mathrm{p}<0.01$ compared with the $0 \mu \mathrm{M}$ icariin-treatment group.

A

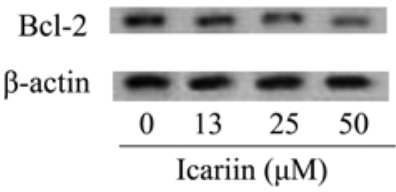

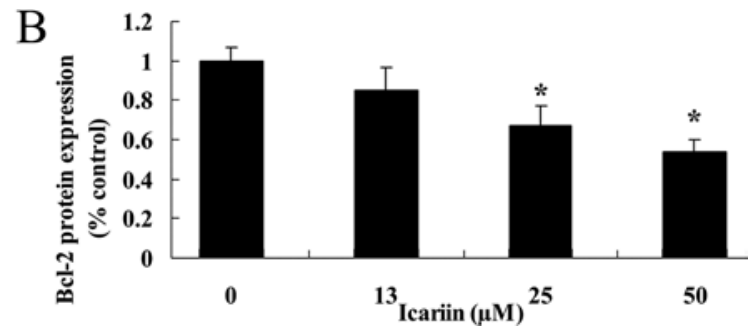

Figure 8. Icariin suppresses Bcl-2 protein expression in the ovarian cancer cells. (A) The effects of icariin on Bcl-2 by western blotting assays. (B) Statistical analysis of the $\mathrm{Bcl}-2$ protein level. ${ }^{\mathrm{p}} \mathrm{p}<0.01$ compared with the $0 \mu \mathrm{M}$ icariin-treatment group.

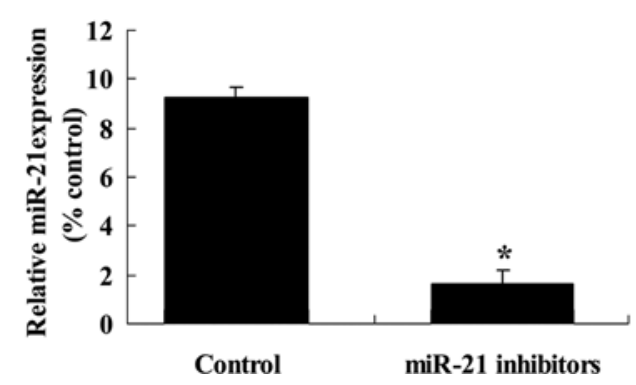

B

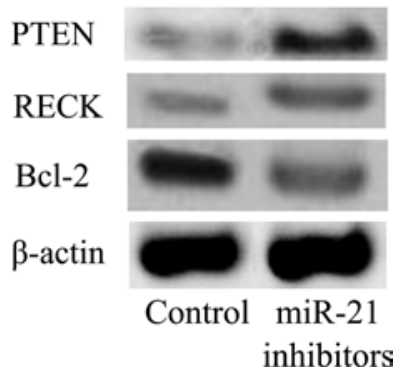

Figure 9. Anti-miR-21 influences expression of PTEN, RECK and Bcl-2 protein in the ovarian cancer cells. (A) Anti-miR-21 influences miR-21 expression. (B) Expression of PTEN, RECK and Bcl-2 protein expression in the A2780 cells. " $\mathrm{p}<0.01$ compared with the $0 \mu \mathrm{M}$ icariin-treatment group.

significantly increased the expression level of miR-21 in the A2780 cells (Fig. 10A). miR-21 mimics reversed the potential effect of icariin on A2780 cell proliferation and reduced activation of caspase-3 (Fig. 10B and C). Moreover, PTEN and RECK protein expression levels were decreased in the A2780 cells (Fig. 10D). In contrast, the $\mathrm{Bcl}-2$ protein expression level in the A2780 cells was increased (Fig. 10D).

\section{Discussion}

Malignant ovarian tumor is one of the three most common female genital cancers. During the past two decades, the application of effective chemotherapy has significantly improved the treatment of malignant ovarian germ cell tumors, with a decline in the mortality rate from 90 to $10 \%$ (25). However, 

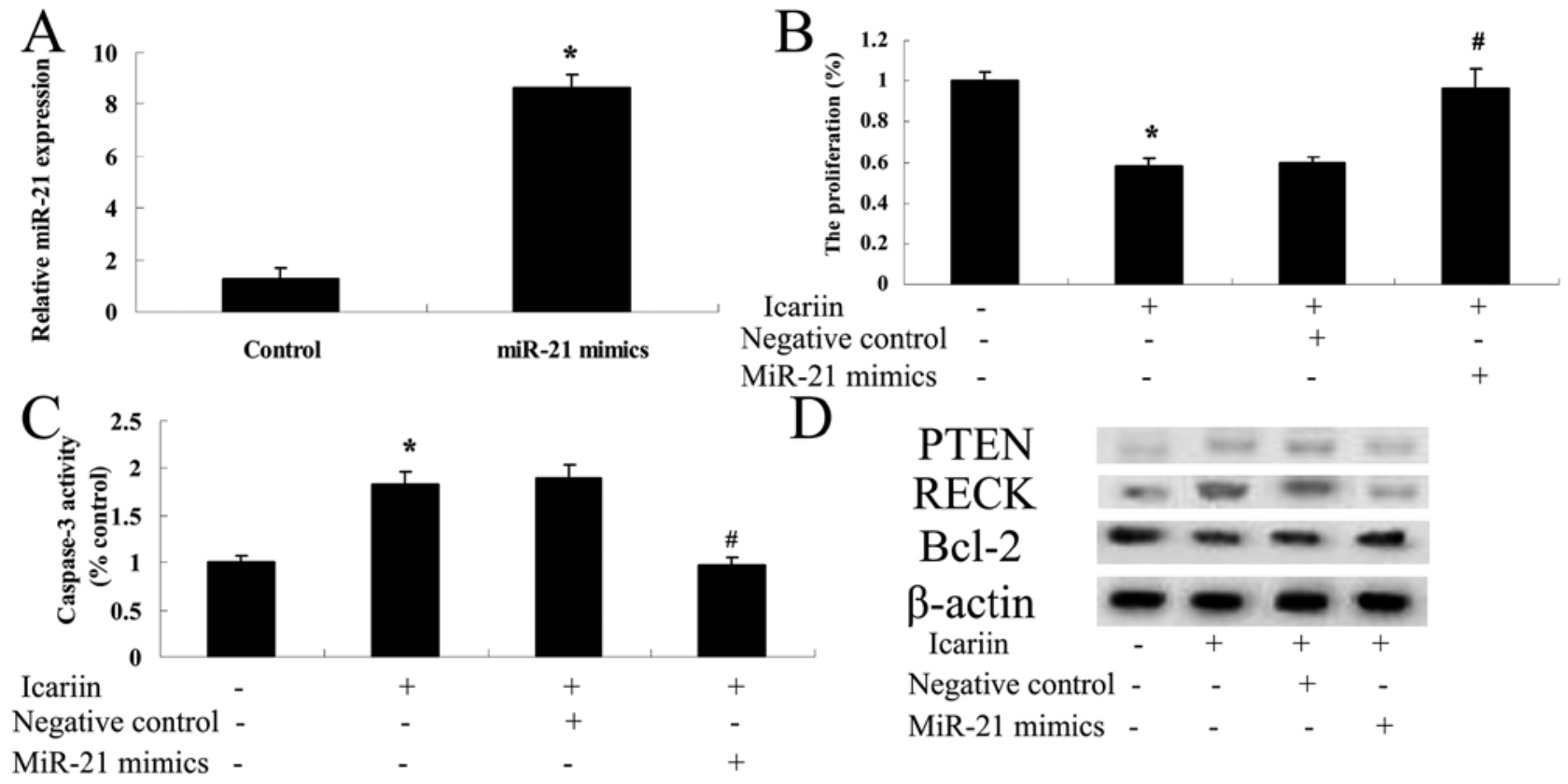

Figure 10. Overexpression of miR-21 influences the effect of icariin on ovarian cancer cells. (A) Transfection of miR-21 significantly increased the expression level of miR-21 in the A2780 cells (B) Following treatment with icariin $(25 \mu \mathrm{M})$ for $48 \mathrm{~h}$, miR-21 mimics promoted the cell proliferation of A2780 cells and (C) reduced activation of caspase-3. (D) Following treatment with icariin $(25 \mu \mathrm{M})$ for $48 \mathrm{~h}$, miR-21 mimics decreased the PTEN and RECK protein expression levels in the A2780 cells while the Bcl-2 protein expression level in the A2780 cells was increased. " $\mathrm{p}<0.01$ compared with the $0 \mu \mathrm{M}$ icariin-treatment group; $" \mathrm{p}<0.01$ compared with the icariin-treatment group transfected with the negative control.

the therapeutic effect of malignant ovarian epithelial tumors has not improved. Malignant ovarian epithelial cancer has become one of the major gynecological cancers, seriously threatening the health and lives of females (26). In the present study, icariin reduced the cell proliferation of human ovarian cancer A2780 cells in a dose- and time-dependent manner. Meanwhile, icariin induced cell apoptosis and enhanced caspase- 3 activity in the A2780 cells. Our results were consistent with those of other studies. For example, Li et al found that icariin induced apoptosis of human hepatoma cells via an ROS/JNK-dependent mitochondrial pathway (27). Yang et al found that icariin exhibited antitumor activity in glioblastoma by inhibiting $\mathrm{NF}-\kappa \mathrm{B}$ activity (22). Zhang et al reported that icariin suppressed cell proliferation, induced apoptosis, and enhanced caspase-3 activity of gallbladder cancer through suppression of the expression of Bcl-2 (28).

Recent studies have shown that miRs play an important role in the occurrence and development of tumors through interaction with mRNAs, involved in proliferation, differentiation, apoptosis and metastasis processes of tumor cells (29). miR-21 has been identified as an oncogene, and is abnormally highly expressed in a variety of tumor tissues through the regulation of tumor-suppressor genes such as NFIB, PDCD4, PTEN and SPRY2 (30). The present study showed that icariin increased the miR-21 expression level in ovarian cancer A2780 cells. Zhang et al reported that icariin adjusted miR-21 in mouse preimplantation embryos (31). The present study for the first time investigated the role of miR-21 in the anticancer effects of icariin on ovarian cancer cells. However, the particular mechanisms of how icariin influences miR-21 expression are still unclear, and further clarification is need.

Low expression of PTEN protein is involved in evolutionary processes of multiple malignant tumors such as prostate and endometrial cancer (32). Studies have shown that PTEN inhibits the motility of tumor cells and angiogenesis by regulating matrix metalloproteinases and vascular endothelial growth factors; moreover, PTEN can dephosphorylate focal adhesion kinase, in which the latter downregulates the MAPK pathway of mitogen-activated protein kinase, thereby regulating cell adhesion (33). Consistently, we found that icariin effectively increased PTEN protein expression in the ovarian cancer A2780 cells. Our results were in keeping with those of other studies. For example, Zhang et al reported that icariin adjusted PTEN protein expression of mouse preimplantation embryos (31). However, the detailed mechanisms on how icariin induces PTEN protein expression in A2780 cells are quite unclear, and further explanation is needed.

RECK protein in patients with deep infiltrative tongue cancer, and in patients with lymph node metastasis is decreased. Thus, RECK protein is considered as an important regulatory protein in inhibiting tumor cell invasion and metastasis. A low level of expression increased the rates of tongue cancer invasion and metastasis, and the regulation may be related to an inhibitory mechanism of RECK on MMP-2 and MMP-9 expression (34). RECK shows an obviously negative correlation with MMP-2 and MMP-9 expression, suggesting that in the process of tumor invasion and metastasis, the expression of RECK is downregulated, limiting the inhibition of RECK on MMP-2 and MMP-9, thus achieving invasion and metastasis of tumor tissues (35). The present study first demonstrated that icariin increased the RECK protein expression level in ovarian cancer A2780 cells. This result demonstrated the potential connection between the anticancer effects of icariin on cell apoptosis and RECK protein expression. Thus, the specific mechanisms of how icariin induces RECK protein expression in A2780 cells remain unclear and further explanation is needed. 
Apoptosis is a form of programmed cell death regulated by genes. Malignant tumors often exhibit increased proliferation and decreased apoptosis. The Bcl-2 family is a group of genes controlling apoptosis, and the $\mathrm{Bcl}-2$ gene has an anti-apoptotic effect (36). Under normal circumstances, Bcl-2 shows cyclical low level expression; yet overexpression of $\mathrm{Bcl}-2$ can inhibit apoptosis. The Bax gene belongs to the same family of $\mathrm{Bcl}-2$ genes, with an opposite function of Bcl-2. Bax promotes apoptosis (37). The dynamic equilibrium between Bcl-2 and Bax is the main mechanism in the regulation of normal apoptosis, and recently many researchers have observed that the level of $\mathrm{Bcl}-2$ protein is related to the sensitivity and resistance of ovarian tumor cells to chemotherapy (38). In the present study, we demonstrated that the $\mathrm{Bcl}-2$ protein expression level was decreased by icariin. This result was in accordance with the findings of other studies. For example, Wang et al reported that icariin induced apoptosis through regulation of the expression of Bcl-2/Bax, and activation of caspase-9 and -3 in mouse MLTC-10 Leydig tumor cells (24). Li et al found that icariin triggered the mitochondrial/caspase-mediated apoptosis of human hepatoma SMMC-7721 cells by suppression of the Bcl-2/Bax ratio (27).

To illuminate the mechanism involved in the suppression of A2780 cell proliferation, the effect of icariin on miR-21 and its target genes, including PTEN, RECK and Bcl-2 was assessed in the A2780 cells. We first found that the miR-21 inhibitor increased PTEN and RECK protein expression levels and decreased the Bcl-2 protein expression level in the ovarian cancer A2780 cells. Then, overexpression of miR-21 suppressed the PTEN and RECK protein expression levels in the A2780 cells. Notably, our data revealed the positive regulation of miR-21, yet negative regulation of the anticancer effect of icariin on cell proliferation and apoptosis in the A2780 cells. These results were in accord with that of other studies. For example, Xu et al demonstrated that suppression of miR-21 decreased cell proliferation and enhanced apoptosis of lung carcinoma by increasing PTEN and RECK and decreasing Bcl-2 mRNA expression (39). The accurate mechanism of how it occurs needs to be further studied.

In summary, the results of the present study further showed the potential anticancer curative effect of icariin on ovarian cancer, and its regulatory mechanism of miR-21 subsequently targeting PTEN, RECK and Bcl-2 in A2780 cells. In particular, our findings revealed that upregulation of miR-21 promoted the progression of ovarian cancer cells through negative regulation of PTEN and RECK expression levels and positive regulation of $\mathrm{Bcl}-2$ expression. We propose that the potential anticancer curative effect of icariin is through the reduction in cell proliferation and promotion of apoptosis through miR-21 and its target genes (PTEN, RECK and Bcl-2) in A2780 cells. We need to further study the molecular mechanisms of icariin on tumorigenesis and progression.

\section{References}

1. Luo J, Zhou J, Cheng Q, Zhou C and Ding Z: Role of microRNA133a in epithelial ovarian cancer pathogenesis and progression. Oncol Lett 7: 1043-1048, 2014.

2. Tang AQ, Cao XC, Tian L, He L and Liu F: Apigenin inhibits the self-renewal capacity of human ovarian cancer SKOV3-derived sphere-forming cells. Mol Med Rep 11: 2221-2226, 2015.
3. Hays JL, Kim G, Walker A, Annunziata CM, Lee JM, Squires J, Houston N, Steinberg SM and Kohn EC: A phase II clinical trial of polyethylene glycol-conjugated L-asparaginase in patients with advanced ovarian cancer: Early closure for safety. Mol Clin Oncol 1: 565-569, 2013.

4. Chen WC, Lin MS, Ye YL, Gao HJ, Song ZY and Shen XY: microRNA expression pattern and its alteration following celecoxib intervention in human colorectal cancer. Exp Ther Med 3: 1039-1048, 2012.

5. Jiang JX, Zhang N, Liu ZM and Wang YY: Detection of microRNA-21 expression as a potential screening biomarker for colorectal cancer: A meta-analysis. Asian Pac J Cancer Prev 15: 7583-7588, 2014

6. Chan JK, Blansit K, Kiet T, Sherman A, Wong G, Earle C and Bourguignon LY: The inhibition of miR-21 promotes apoptosis and chemosensitivity in ovarian cancer. Gynecol Oncol 132: 739-744, 2014.

7. Liu S, Fang Y, Shen $\mathrm{H}, \mathrm{Xu} \mathrm{W}$ and Li H: Berberine sensitizes ovarian cancer cells to cisplatin through miR-21/PDCD4 axis. Acta Biochim Biophys Sin 45: 756-762, 2013.

8. Yang L, Kuang LG, Zheng HC, Li JY, Wu DY, Zhang SM and Xin Y: PTEN encoding product: A marker for tumorigenesis and progression of gastric carcinoma. World J Gastroenterol 9: 35-39, 2003.

9. Wu H, Wang K, Liu W and Hao Q: PTEN overexpression improves cisplatin-resistance of human ovarian cancer cells through upregulating KRT10 expression. Biochem Biophys Res Commun 444: 141-146, 2014.

10. Lau MT, Klausen $C$ and Leung PC: E-cadherin inhibits tumor cell growth by suppressing PI3K/Akt signaling via $\beta$-cateninEgrl-mediated PTEN expression. Oncogene 30: 2753-2766, 2011.

11. Lou Y, Yang X, Wang F, Cui Z and Huang Y: MicroRNA-21 promotes the cell proliferation, invasion and migration abilities in ovarian epithelial carcinomas through inhibiting the expression of PTEN protein. Int J Mol Med 26: 819-827, 2010.

12. Zhou X, Huang S, Jiang L, Zhang S, Li W, Chen Z and Zhang D: Expression of RECK and MMP-2 in salivary adenoid cystic carcinoma: Correlation with tumor progression and patient prognosis. Oncol Lett 7: 1549-1555, 2014.

13. Xue G, Zou X, Zhou JY, Sun W, Wu J, Xu JL and Wang RP: Raddeanin A induces human gastric cancer cells apoptosis and inhibits their invasion in vitro. Biochem Biophys Res Commun 439: 196-202, 2013.

14. Masui T, Doi R, Koshiba T, Fujimoto K, Tsuji S, Nakajima S, Koizumi M, Toyoda E, Tulachan S, Ito D, et al: RECK expression in pancreatic cancer: Its correlation with lower invasiveness and better prognosis. Clin Cancer Res 9: 1779-1784, 2003.

15. Zhang Y, Cheng S, Zhang G, Ma W, Liu Y, Zhao R, Zhang Q and Pang D: Low expression of RECK indicates a shorter survival for patients with invasive breast cancer. Cancer Sci 103: 1084-1089, 2012.

16. Qi Q, Lu N, Li C, Zhao J, Liu W, You Q and Guo Q: Involvement of RECK in gambogic acid induced anti-invasive effect in A549 human lung carcinoma cells. Mol Carcinog: Feb 14, 2014 (Epub ahead of print). doi: $10.1002 / \mathrm{mc} .22138$.

17. Fejzo MS, Ginther C, Dering J, Anderson L, Venkatesan N, Konecny G, Karlan B and Slamon DJ: Knockdown of ovarian cancer amplification target $A D R M 1$ leads to downregulation of GIPCI and upregulation of RECK. Genes Chromosomes Cancer 50: 434-441, 2011.

18. Ahn MY, Kang DO, Na YJ, Yoon S, Choi WS, Kang KW, Chung HY, Jung JH, Min do S and Kim HS: Histone deacetylase inhibitor, apicidin, inhibits human ovarian cancer cell migration via class II histone deacetylase 4 silencing. Cancer Lett 325: 189-199, 2012.

19. Sadahira K, Sagawa M, Nakazato T, Uchida H, Ikeda Y, Okamoto S, Nakajima $\mathrm{H}$ and Kizaki M: Gossypol induces apoptosis in multiple myeloma cells by inhibition of interleukin-6 signaling and Bcl-2/Mcl-1 pathway. Int J Oncol 45: 2278-2286, 2014.

20. Xie YL, Yang YJ, Tang C, Sheng HJ, Jiang Y, Han K and Ding LJ: Estrogen combined with progesterone decreases cell proliferation and inhibits the expression of Bcl-2 via microRNA let-7a and miR-34b in ovarian cancer cells. Clin Transl Oncol 16: 898-905, 2014

21. Ma C, Yin G, You F, Wei Y, Huang Z, Chen X and Yan D: A specific cell-penetrating peptide induces apoptosis in SKOV3 cells by down-regulation of Bcl-2. Biotechnol Lett 35: 1791-1797, 2013. 
22. Yang L, Wang Y, Guo H and Guo M: Synergistic anti-cancer effects of icariin and temozolomide in glioblastoma. Cell Biochem Biophys: Nov 11, 2014 (Epub ahead of print).

23. Zhou J, Wu J, Chen X, Fortenbery N, Eksioglu E, Kodumudi KN, Pk EB, Dong J, Djeu JY and Wei S: Icariin and its derivative, ICT, exert anti-inflammatory, anti-tumor effects, and modulate myeloid derived suppressive cells (MDSCs) functions. Int Immunopharmacol 11: 890-898, 2011.

24. Wang Q, Hao J, Pu J, Zhao L, Lü Z, Hu J, Yu Q, Wang Y, Xie Y and Li G: Icariin induces apoptosis in mouse MLTC-10 Leydig tumor cells through activation of the mitochondrial pathway and down-regulation of the expression of piwil4. Int J Oncol 39: 973-980, 2011

25. Nakamura K, Banno K, Yanokura M, Iida M, Adachi M, Masuda K, Ueki A, Kobayashi Y, Nomura H, Hirasawa A, et al: Features of ovarian cancer in Lynch syndrome (Review). Mol Clin Oncol 2: 909-916, 2014.

26. Li S, Li Y, Wen Z, Kong F, Guan X and Liu W: microRNA-206 overexpression inhibits cellular proliferation and invasion of estrogen receptor $\alpha$-positive ovarian cancer cells. Mol Med Rep 9: 1703-1708, 2014

27. Li S, Dong P, Wang J, Zhang J, Gu J, Wu X, Wu W, Fei X, Zhang Z, Wang Y, et al: Icariin, a natural flavonol glycoside, induces apoptosis in human hepatoma SMMC-7721 cells via a ROS/JNK-dependent mitochondrial pathway. Cancer Lett 298: 222-230, 2010.

28. Zhang DC, Liu JL, Ding YB, Xia JG and Chen GY: Icariin potentiates the antitumor activity of gemcitabine in gallbladder cancer by suppressing NF- $\mathrm{BB}$. Acta Pharmacol Sin 34: 301-308, 2013.

29. Wang B and Zhang Q: The expression and clinical significance of circulating microRNA-21 in serum of five solid tumors. J Cancer Res Clin Oncol 138: 1659-1666, 2012.

30. Liu M, Tang Q, Qiu M, Lang N, Li M, Zheng Y and Bi F: miR-21 targets the tumor suppressor RhoB and regulates proliferation, invasion and apoptosis in colorectal cancer cells. FEBS Lett 585: 2998-3005, 2011.

31. Zhang C, Shi YR, Liu XR, Cao YC, Tian JL, Jia ZY, Zhen D, Liu FH and Gao JM: The regulatory role of icariin on apoptosis in mouse preimplantation embryos with reduced microRNA-21. Theriogenology 82: 461-468, 2014.
32. Waite KA and Eng C: Protean PTEN: Form and function. Am J Hum Genet 70: 829-844, 2002.

33. Gu J, Tamura M and Yamada KM: Tumor suppressor PTEN inhibits integrin- and growth factor-mediated mitogen-activated protein (MAP) kinase signaling pathways. J Cell Biol 143: 1375-1383, 1998

34. Alexius-Lindgren M, Andersson E, Lindstedt I and Engström W: The RECK gene and biological malignancy - its significance in angiogenesis and inhibition of matrix metalloproteinases. Anticancer Res 34: 3867-3873, 2014.

35. Prosdócimi FC, Rodini CO, Sogayar MC, Sousa SC, Xavier FC and Paiva KB: Calcifying cystic odontogenic tumour: Immunohistochemical expression of matrix metalloproteinases, their inhibitors (TIMPs and RECK) and inducer (EMMPRIN). J Oral Pathol Med 43: 545-553, 2014.

36. Liu N, Zheng Y, Zhu Y, Xiong S and Chu Y: Selective impairment of $\mathrm{CD} 4^{+} \mathrm{CD} 25^{+} \mathrm{Foxp}^{+}$regulatory T cells by paclitaxel is explained by $\mathrm{Bcl}-2 / \mathrm{Bax}$ mediated apoptosis. Int Immunopharmacol 11: 212-219, 2011.

37. Li J, Sun GZ, Lin HS, Pei YX, Qi X, An C, Yu J and Hua BJ: The herb medicine formula 'Yang Wei Kang Liu' improves the survival of late stage gastric cancer patients and induces the apoptosis of human gastric cancer cell line through Fas/Fas ligand and Bax/Bcl-2 pathways. Int Immunopharmacol 8: 1196-1206, 2008.

38. Yao Y, Huang C, Li ZF, Wang AY, Liu LY, Zhao XG, Luo Y, Ni L, Zhang WG and Song TS: Exogenous phosphatidylethanolamine induces apoptosis of human hepatoma HepG2 cells via the bcl-2/Bax pathway. World J Gastroenterol 15: 1751-1758, 2009.

39. Xu LF, Wu ZP, Chen Y, Zhu QS, Hamidi S and Navab R: MicroRNA-21 (miR-21) regulates cellular proliferation, invasion, migration, and apoptosis by targeting PTEN, RECK and $\mathrm{Bcl}-2$ in lung squamous carcinoma, Gejiu City, China. PLoS One 9: e103698, 2014. 